\title{
CORRESPONSABILITÀ, AMMINISTRAZIONE E SOSTEGNO ECONOMICO ALLA CHIESA: VERSO UNA "STEWARDSHIP" EUROPEA? *
}

Sommario: - 1. L'idea di "stewardship". - 2. Il contenuto del documento americano. - 3. Contestualizzazione ecclesiologica della nozione di "stewardship". 4. Contestualizzazione storica del documento americano sulla "stewardship". - 5. Due premesse per la comprensione della "stewardship". - 6. La "stewardship" nel contesto del sostegno economico pubblico alla Chiesa. - Conclusione.

In tema di finanziamento delle confessioni religiose da parte delle autorità civili, il riferimento alla "stewardship", scelta come argomento di questo lavoro, può destare meraviglia. Che significato può avere un discorso sulla corresponsabilità in un contesto di finanziamento "extra-ecclesiale", o se si preferisce "extra-confessionale", delle confessioni religiose? Penso che in una corretta visione dei rapporti tra le confessioni e le autorità civili, in una prospettiva che parta dalla consapevolezza che i fedeli sono anche cittadini o che i cittadini sono anche fedeli, e che pertanto il bene dei fedeli é anche il bene dei cittadini, in un contesto civico che valorizzi il bene comune...; in una visione di questo tipo, il discorso sulla corresponsabilità può avere un significato anche nell'ambito del finanziamento pubblico delle confessioni religiose.

In realtà, il titolo di questo intervento richiama una riflessione più ampia e riprende il nome di un gruppo di ricerca e di scambio di idee

* Il testo provience dal contributo al Convegno "Il finanziamento pubblico della Chiesa: sguargi incrociati tra Svizzera ed Europa", organizzato dall'Istituto Internazionale di Diritto canonico e diritto comparato delle religioni (DIRECOM) e dalla Facoltà di Teologia di Lugano (Svizzera), 13-14 settembre 2012. 
nato nel 2011 a Roma in una riunione di canonisti, pastoralisti e comunicatori interessati appunto alle tematiche che riguardano il sostegno economico della Chiesa cattolica. Il gruppo è stato chiamato CASE (per le iniziali di corresponsabilità, amministrazione e sostegno economico $)^{1}$ e si è proposto come primo traguardo quello di studiare le possibilità di importare in altri ambiti culturali l'esperienza della "stewardship" negli Stati Uniti di America ${ }^{2}$. Ma 1'ampiezza della riflessione indicata dal titolo non esclude la sua adeguata applicazione al concreto ambito del finanziamento pubblico delle confessioni religiose. Cercherò di presentare qui alcuni spunti di studio in tal senso.

\section{L'idea di "stewardship"}

"Stewardship" e "steward" sono i termini adoperati dalle traduzioni inglesi della Sacra Scrittura per riportare quello che in italiano viene tradotto come "amministrazione" e "amministratore"3. Nel Nuovo Testamento è usato anche per la traduzione del passo della prima lettera di Pietro (1Pt 4,10): «Ciascuno viva secondo la grazia ricevuta, mettendola a servizio degli altri, come buoni amministratori di una multiforme grazia di Dio» ${ }^{4}$. La Conferenza episcopale degli Stati Uniti d'America aprì con questa citazione un documento ("pastoral letter") intitolato "Stewardship: A Disciple's Response"5, pubblicato nel 1992, che ha determi-

1 Cfr. www.casestewardship.org.

${ }^{2}$ Un primo risultato del lavoro di CASE è la pubblicazione di un gruppo di tre lavori sull'argomento raccolti nelle pagine di Ius Ecclesiae 24(2012) 277-323: J. MiñAmBrES, La 'stewardship' (corresponsabilità) nella gestione dei beni temporali della Chiesa; M. Rivella, Buon governo e corresponsabilità; D. ZALBIDEA, Corresponsabilidad (stewardship) y derecho canónico.

3 Vedi, ad esempio, Lc 12,42-43: «Qual è dunque l'amministratore [steward] fedele e saggio, che il Signore porrà a capo della sua servitù, per distribuire a tempo debito la razione di cibo? Beato quel servo che il padrone, arrivando, troverà al suo lavoro»; $L c$ 16,1: «Diceva anche ai discepoli: “C'era un uomo ricco che aveva un amministratore [steward], e questi fu accusato dinanzi a lui di sperperare i suoi averi”»; ecc.

${ }^{4}$ In inglese: «As each one has received a gift, use it to serve one another as good stewards of God's varied grace».

${ }^{5}$ USCCB, Stewardship: A Disciple's Response, Tenth Anniversary Edition, Washington, DC 2002, in http://usccb.org/stewardship/disciplesresponse.pdf. 
nato un movimento di rinnovamento della comunione nella Chiesa degli Stati Uniti, e ha portato ad una nuova comprensione di quell' aspetto della comunione che viene abitualmente denominato "corresponsabilità"6.

La nozione di "stewardship" proposta dai Vescovi americani non è nuova; era già stata studiata dagli economisti per la "governance" delle organizzazioni dette di "missione", soprattutto del terzo settore ("nonprofit": volontariato, cooperative, ecc.), ma anche statali o governative ed altre. Si tratta di entità che non cercano di costruire un prodotto o di erogare un servizio ma si propongono una "missione" (proteggere l'ambiente, accogliere gli immigranti, ecc.). Viene adoperata anche nel mondo del "fund raising" per il coinvolgimento dei donatori nel programma dell'impresa finanziata ${ }^{8}$. E ricorre anche in ecologia come richiesta di responsabilizzazione degli esseri umani nell'uso delle risorse della natura?

I Vescovi americani riprendono il concetto ("stewardship") e lo applicano nella Chiesa con gli adattamenti del caso. Ottengono in tal modo una comprensione del problema del finanziamento delle attività necessarie per l'evangelizzazione e la missione della Chiesa che arriva più alla radice: la responsabilità di tutti i fedeli cattolici nei confronti dell'intera vita della Chiesa, quindi anche nel ritrovamento delle risorse materiali per poter affrontare l'evangelizzazione e la missione. Così, i Vescovi americani arrivano alla conclusione che il finanziamento delle diverse attività ecclesiali non è "il" problema, ma soltanto una parte di esso (in realtà molto piccola e poco rilevante). Il vero "problema" è

\footnotetext{
${ }^{6}$ Chiariamo che noi abbiamo mantenuto spesso l'uso della parola inglese "stewardship" anziché di un termine italiano per la difficoltà di tradurre con un solo vocabolo italiano tutto il contenuto del concetto compreso nella parola inglese, che riguarda certamente l'amministrazione, ma anche, come appena detto, la corresponsabilità, e poi la trasparenza, la partecipazione, ecc., e anche la risultante dalla combinazione di tutti questi elementi insieme.

${ }^{7}$ Cfr., ad esempio, P. C. Brinckerhoff, Nonprofit Stewardship. A Better Way to Lead Your Mission-Based Organization, Saint Paul (MN) 2004.

${ }^{8}$ Cfr., ad es., R.D. Barret-M.E. WAre, Planned Giving Essentials: A Step-by-Step Guide to Success, Gaithersburg 2002 (2nd ed.).

9 Cfr., ad es., T. Whitmore-M.A. Ryan, The Challenge of Global Stewardship. Roman Catholic Responses, Notre Dame (IN) 1997.
} 
la crescita dei fedeli, la conversione personale, la ricerca della santità; il tutto nel contesto della comunione della Chiesa. In questo senso, il maggiore coinvolgimento dei fedeli nella vita della Chiesa in generale comporta anche una loro maggiore responsabilità economica.

Tale maggiore coinvolgimento pensato dai Vescovi americani non consiste nello svolgimento di più funzioni, nel ricoprire più cariche $\mathrm{o}$ nel partecipare a più riunioni, per fare soltanto alcuni esempi, ma è il frutto della conversione personale, della santità di ciascuno. E così, l'idea della "stewardship" viene ad essere concepita come un modo di attuare la risposta personale del discepolo di Cristo al suo Maestro ("a disciple's response", appunto). Certamente, i Vescovi non perdono di vista l'importanza di sovvenire alle necessità materiali della Chiesa, ma collocano tale esigenza nel contesto più ampio della condizione di cristiano ${ }^{10}$. In questa visione, $i$ Vescovi compiono il loro dovere ${ }^{11}$ di ricordare a tutti i fedeli l'obbligo «di sovvenire alle necessità della Chiesa» secondo quanto stabilito dal can. $222 \S 1 \mathrm{CIC}^{12}$, ma lo fanno con una motivazione molto più comprensibile di altre adoperate frequentemente. Ad esempio, quando l'obbligo di sovvenire alle necessità materiali della Chiesa viene fatto dipendere esclusivamente dalla sua doppia natura (spirituale e umana), seguendo quanto ricorda Lumen gentium (n. 8), si fa perno sul bisogno di adoperare "cose materiali" e non sulla responsabilità dei fedeli di procurarle. Invece, quando la motivazione poggia sulla "stewardship" non si chiede di fare qualcosa per qualcuno che è nel bisogno (per la Chiesa), ma si chiede piuttosto di crescere come fedele e discepolo di Cristo; non di fare, ma di essere.

\footnotetext{
${ }^{10}$ Così si esprime l'introduzione al documento: «This pastoral letter recognizes the importance of church support, including the sharing of time, talent and treasure. But it situates church support in its broader context - what it means to be a disciple of Christ» (loc. cit., p. 6).

${ }^{11}$ Can. 1261 § 2. «Il Vescovo diocesano è tenuto ad ammonire i fedeli sull'obbligo di cui al can. 222, $§ 1$, urgendone l'osservanza in maniera opportuna».

${ }^{12}$ «I fedeli sono tenuti all'obbligo di sovvenire alle necessità della Chiesa, affinché essa possa disporre di quanto è necessario per il culto divino, per le opere di apostolato e di carità e per l'onesto sostentamento dei ministri».
} 
La "stewardship" viene presentata quindi come parte della risposta del discepolo di Cristo. Essa ha una dimensione personale che mette in atto l'iniziativa dei singoli fedeli ${ }^{13}$, e anche una dimensione comunionale, che viene vista nel documento soprattutto in riferimento alla famiglia, "chiesa domestica", alla parrocchia e alla diocesi ${ }^{14}$, anche se gli sviluppi successivi dell'idea di "stewardship" e le sue realizzazioni si sono concentrati principalmente in questi ultimi due ambiti comunitari all'interno della Chiesa: la parrocchia e la diocesi.

Interessa ora esaminare soprattutto le ricadute giuridiche che una tale concezione dell'obbligo dei fedeli di sovvenire alle necessità materiali della Chiesa possa avere sul sostegno economico alla stessa. Logicamente, un maggiore senso di appartenenza dei fedeli favorirà senz'altro i contributi volontari, spontanei e su richiesta ${ }^{15}$, e potrebbe anche migliorare la comprensione degli altri mezzi stabiliti dalla legislazione canonica codiciale vigente, dalle tasse ai tributi, senza dimenticare le "volontà pie" sia tra vivi che mortis causa. Ma, come dicevamo prima, se ci si ferma a pensare un poco più a lungo la "stewardship" può modificare anche la comprensione dei rapporti con le autorità civili (statali, cantonali, locali...).

\section{Il contenuto del documento americano}

La "pastoral letter" dei vescovi americani si apre con una introduzione nella quale si ricordano alcuni convincimenti che sottostanno al documento: 1. La maturità cristiana si mostra nella decisione ferma e consapevole di seguire Cristo ad ogni costo; 2 . La conversione e la dedizione cristiane si manifestano lungo tutta la vita e non soltanto in singoli atti; 3. La "stewardship" è un'espressione del discepolato cristiano che parte dal riconoscimento di Dio come datore di ogni bene e dal ringraziamento che si manifesta nel far fruttare i doni ricevuti

${ }^{13}$ Cfr. p. 32 del documento: «(...) members of the Church (...) should engage in such activities personally and on their own initiative».

${ }^{14}$ Cfr. ibid.

${ }^{15}$ Cfr. J.-P. Schouppe, Elementi di diritto patrimoniale canonico, Milano $2008\left(2^{\circ}\right.$ ed.), p. 85-118. 
per amore a Dio e agli altri ${ }^{16}$. I vescovi propongono, poi, la sfida di coltivare la coerenza cristiana in una cultura caratterizzata da materialismo, relativismo, edonismo, individualismo e consumismo ${ }^{17}$. Per poter accogliere la sfida occorre poggiare sulla generosità nella condivisione dei doni ricevuti; e in questo ambito bisogna introdurre anche il sostentamento economico alla Chiesa, ma nel contesto più ampio della condivisione generosa e responsabile di uno stile di vita radicato nel sapersi discepoli di Cristo $^{18}$. L'introduzione finisce con una chiamata ad applicare questa visione alle circostanze particolari degli Stati Uniti d'America negli ultimi anni del ventesimo secolo ${ }^{19}$.

Il documento americano è diviso in cinque parti. Inizia dalla vocazione (The $\mathrm{Call}^{20}$ ) e, dopo aver offerto una considerazione cristologica (Jesus' $W a y^{21}$ ), si sofferma sulla presentazione dei modi di vivere come buoni amministratori del creato e cooperatori della redenzione (Living as a Steward ${ }^{22}$ ). Prende poi in esame 1'effetto di quanto spiegato sui modi di vivere la comunione nella Chiesa (Stewards of the Church ${ }^{23}$ ) e finisce con un ritratto del singolo cristiano che si sforza di vivere secondo questo schema (The Christian Steward ${ }^{24}$ ).

Come abbiamo più volte ripetuto, tratto caratteristico della "pastoral letter" è quello di rivolgersi ai cattolici degli Stati Uniti di America. Certamente, le condizioni culturali, storiche ed esistenziali (anche economiche) dei cattolici europei sono molto diverse. Si potrebbe quindi pensare che il documento dei Vescovi americani non meriti particolare considerazione da parte degli studiosi e dei pastori di questa parte dell'Atlantico. Per questo motivo, il titolo di questo lavoro finisce con un punto interrogativo: "verso una stewardship europea?" Personalmente

\footnotetext{
${ }^{16}$ Cfr. USCCB, Stewardship, p. 5.

${ }^{17}$ Cfr. ibid.

${ }^{18}$ Cfr. ibid., p. 6-7.

${ }^{19}$ Cfr. ibid., p. 8.

${ }^{20}$ Ibid., p. 13-17.

${ }^{21}$ Ibid., p. 19-23.

${ }^{22}$ Ibid., p. 25-30.

${ }^{23}$ Ibid., p. 31-37.

${ }^{24}$ Ibid., p. 39-41.
} 
sono convinto che il documento americano possa offrire riferimenti validi per l'intera Chiesa e, perciò, anche per i cattolici europei. In fondo, non fa altro che rendere esplicite alcune esigenze della vita cristiana, della comunione nella Chiesa, che riguardano l'obbligo di sostenerla nei suoi bisogni materiali (cfr. can. $222 \mathrm{CIC}$ ). Comunque, più avanti accenneremo a diversi documenti europei più o meno contemporanei di quello americano che affrontano anche la materia in termini simili.

\section{Contestualizzazione ecclesiologica della nozione di "stewardship"}

Il documento sulla "stewardship" e le idee in esso espresse si inseriscono in un contesto di comunione, della Chiesa intesa come comunione. Il riferimento alla comunione ecclesiale è particolarmente opportuno in questo tempo ${ }^{25}$. Il Beato Giovanni Paolo II l'ha riproposta a tutti i fedeli come nozione centrale all'inizio del terzo millennio: «Fare della Chiesa la casa e la scuola della comunione: ecco la grande sfida che ci sta davanti nel millennio che inizia, se vogliamo essere fedeli al disegno di Dio e rispondere anche alle attese profonde del mondo ${ }^{26}$.

In effetti, la comunione è l'ambito proprio di sviluppo della corresponsabilità di tutti i fedeli nella costruzione e nella missione della Chiesa ed è uno degli sviluppi principali apportati dal Concilio Vaticano II in materia di ecclesiologia: il Card. Suenens, che ebbe un ruolo assai rilevante nei lavori conciliari, chiamò la corresponsabilità «idea maestra del Concilio» ${ }^{27}$. In questo senso, la descrizione del-

${ }^{25}$ In realtà è il modo adeguato di descrivere l'essenza stessa della Chiesa (cfr. CoNgregazione per la Dottrina della Fede, Communionis notio, 28 maggio 1992, AAS 85(1993) 838-850, n. 1). L'argomento è stato spesso studiato anche dal punto di vista prettamente giuridico; oltre agli studi di Corecco che menzioneremo nel testo, può essere utile, ad esempio, W. Aymans, Diritto canonico e comunione ecclesiale. Saggi di diritto canonico in prospettiva teologica, Torino 1993; P.A. Bonnet, Comunione ecclesiale e diritto, Monitor ecclesiasticus 116(1991) 49-86; A. MARzoA, Comunión y derecho: significación e implicaciones de ambos conceptos, Pamplona 1999.

${ }^{26}$ Giovanni Paolo II, Lett. Ap. Novo millennio ineunte, 6 gennaio 2001 (AAS 93 [2001] 266-309), n. 43.

${ }^{27}$ L.J. Suenens, La corresponsabilité dans l'Église d'ajourd'hui, Paris 1968, p. 7. 
la Chiesa come famiglia da parte del costituzione dogmatica Lumen gentium (de Ecclesia), cioè il documento che il Concilio ha dedicato proprio a studiare la $\mathrm{Chiesa}^{28}$, diventa particolarmente illuminante per la comprensione dell'aspetto della comunione più sottolineato dalla corresponsabilità. In particolare, per quanto riguarda quell'aspetto della corresponsabilità che si traduce nella consapevolezza del dovere relativo al finanziamento della Chiesa, la figura della famiglia rende evidente la necessità del coinvolgimento di tutti (non c'è famiglia se non ci sono familiari), ma aiuta anche a percepire la necessaria differenziazione dei ruoli (non hanno le stesse responsabilità economiche i genitori o i figli, e vi è anche differenza tra l'impegno che si può chiedere a un figlio di tre anni o a uno di sedici). Dal punto di vista della corresponsabilità ecclesiale che riguarda il sostegno economico, il paragone con la famiglia rende soprattutto palese che i contributi economici alla Chiesa non vanno verso qualcosa di estraneo a me, così come i contributi economici alla famiglia fanno parte della mia vita: donare alla Chiesa parte dello stipendio dovrebbe essere come mettere a disposizione della famiglia quello che si percepisce con il proprio lavoro. Certamente, giacché si tratta di analogia, il paragone non va portato alle estreme conseguenze, anche perché le politiche tributarie dei diversi Paesi non lo permetterebbero, ma può aiutare a chiarire soggettivamente la scelta di apportare beni economici alla comunione della Chiesa: l'appartenenza alla comunità ecclesiale, analogamente a quanto succede con l'appartenenza familiare, si manifesta in tutti gli aspetti della mia vita, anche in quello economico.

${ }^{28} \mathrm{Cfr}$. cost. dogm. Lumen gentium, 6. Il riferimento alla famiglia nel contesto della corresponsabilità di tutti i fedeli e in particolare dei laici è stato ripreso recentemente da Benedetto XVI nel suo Messaggio in occasione della VI assemblea ordinaria del Forum Internazionale di Azione Cattolica, 10 agosto 2012 (pubblicato in www.vatican.va/holy_father/benedict_xvi/messages/pont-messages/2012/documents/hf_benxvi_mes_20120810_fiac_it.html). 


\section{Contestualizzazione storica del documento americano sulla "stewardship"}

In concomitanza con lo sviluppo dottrinale della riflessione sulla comunione ecclesiale e sulle sue conseguenze giuridiche ${ }^{29}$, il Concilio Vaticano II prese una decisione riguardante il sostegno materiale della Chiesa (in realtà, principalmente riguardava il sostentamento del clero) che avrebbe avuto notevoli conseguenze negli anni a venire: la determinazione di abolire, o almeno riformare profondamente, il sistema beneficiale ${ }^{30}$. Le Norme per l'applicazione di alcuni decreti del

${ }^{29}$ Basti ricordare, ad esempio, l'applicazione "costituzionale" della dimensione della Chiesa come comunione che si realizza nel denominato principio di "uguaglianza" (cfr., tra altri, A. Del Portillo, Laici e fedeli nella Chiesa. Le basi dei loro statuti giuridici, Milano 1999 [originariamente pubblicato in spagnolo nel 1967]; J. HERVADA, Diritto costituzionale canonico, Milano 1989, p. 86 ss.; P. Lombardía, Los laicos en el Derecho de la Iglesia, Ius Canonicum 6[1966] 339-374; L. NAVARRo, Il principio costituzionale di uguaglianza nell'ordinamento canonico, Fidelium iura 2[1992] 145-163) e che ha originato molti studi canonistici importanti, anche sulla corresponsabilità di tutti i fedeli (cfr., ad esempio, J. Beyer, G. Felictani, H. Müller, Comunione ecclesiale e strutture di corresponsabilità, Roma 1990; V. Gómez-IGLESIAS C., Alcance canónico de la corresponsabilidad y participación de los fieles en la misión de la Iglesia, Fidelium iura 9[1999] 161-202).

${ }^{30}$ «(...) il sistema detto beneficiale va abbandonato, o almeno riformato in modo che la parte beneficiale —ossia il diritto al reddito di cui è dotato l'ufficio- sia considerata come secondaria, e venga giuridicamente messo in primo piano l'ufficio ecclesiastico» (decr. Presbyterorum Ordinis, n. 20 b). Per la comprensione dell'istituto e della sua storia sono ancora utili M. Petroncelli, Beneficio ecclesiastico (diritto civile), in: Enciclopedia del diritto, V, Milano 1959, p. 131-144; P. Fedele, Beneficio ecclesiastico (diritto canonico), in: Enciclopedia del diritto, V, Milano 1959, p. 144 -156; V. De Reina, El sistema beneficial, Pamplona 1965. La trattazione del beneficio da parte del Concilio e della sua evoluzione posteriore è stata oggetto di abbondanti studi, tra i quali possono essere consultati O. RoBledA, Innovationes Concilii Vaticani II in theoria et disciplina de officiis et beneficiis ecclesiasticis, Periodica 58(1969) 155-198; E. Colagiovanni, L'aspetto storico - sociologico nella trasformazione dal beneficio feudale all 'ufficio ecclesiastico ed ecclesiale, in: AA. VV., Dal beneficio feudale all'ufficio ecclesiastico ed ecclesiale, Napoli 1971, p. 29-54; R. BACCARI, L'aspetto giuridico-pastorale della trasformazione del beneficio in ufficio, Monitor ecclesiasticus 96(1971) 419-431; T. MAURO, Il sistema beneficiale italiano nella prospettiva del nuovo Concordato, in: T. Mauro, Scritti di diritto ecclesiastico e canonico, II, Padova 1991, p. 533-557; A. Vitale, Dai benefici ecclesiastici agli Istituti per il sostentamento 
concilio Vaticano II, pubblicate da Paolo VI il 6 agosto 1966 mediante il motu proprio Ecclesiae Sanctae ${ }^{31}$, optarono per la soluzione più radicale, l'abolizione, confermata poi dal can. 1272 del Codice del 1983. L'abolizione del sistema beneficiale, insieme alla richiesta conciliare di trovare nuovi modi per gli interventi delle autorità civili nella provvista degli uffici ecclesiastici che garantissero meglio la libertà della Chie$\mathrm{sa}^{32}$, diede una spinta per l'instaurazione di una nuova fase "concordataria". In questo contesto si avviò la revisione di diversi concordati (italiano, spagnolo, ecc.) per trovare sistemi di collaborazione nella designazione dei candidati ad alcuni uffici, soprattutto quelli episcopali, e anche per cercare di venire incontro alla necessità di trovare nuove risorse materiali che sostituissero quelle che tradizionalmente apportavano i benefici.

All'interno della gerarchia ecclesiastica, però, il cambiamento auspicato dal documento conciliare, concretizzato nell'abolizione del sistema beneficiale, indusse alcuni vescovi a manifestare una certa preoccupazione per garantire i fondi necessari per portare avanti la missione di quelle strutture pastorali che hanno meno contatto diretto con i fedeli, in modo particolare le diocesi ${ }^{33}$. Per questo motivo, nella redazione dei canoni del CIC venne introdotta la figura dei tributi diocesani (cfr. can. 1263), in qualche modo già presente nell'ordinamento con il nome di "cattedratico" ma che adesso si poneva come fondamentale

del clero, in: AA.VV., Il nuovo regime giuridico degli enti e dei beni ecclesiastici, cur. A. CASIRAghi, Milano 1993, p. 139-157; F. Falchi, Dal beneficio ecclesiastico alla Massa comune dei beni nei dibattiti del Concilio Vaticano II, ibid., p. 227-251; V. DE PAolis, Il sistema beneficiale e il suo superamento dal Concilio Vaticano II ai nostri giorni, in: AA. VV., Il sostentamento del clero, Città del Vaticano 1993, p. 21-31; G. Corbellini, Note sulla formazione del can. 1274 (e dei cann. 1275 e 1272) del "Codex Iuris canonici”, Ius Ecclesiae 8(1996) 465-507.

${ }^{31}$ Cfr. AAS 58(1966) 757-787.

${ }^{32} \mathrm{Ci}$ siamo occupati di questi aspetti in J. MiñamBres, La presentazione canonica. Collaborazione nella provvista degli uffici ecclesiastici, Milano 2000, p. 79 ss.

${ }^{33}$ Interessante in questo senso l'intervento di Bernardin raccolto in Pontificium Consilium de Legum Textibus Interpretandis, Congregatio plenaria diebus 20-29 octobris 1981 habita, Typis Polyglottis Vaticanis 1991, p. 490. 
per garantire la sopravvivenza delle diocesi ${ }^{34}$. Le attese erano quindi contrastanti: ci si auspicava una migliore organizzazione degli uffici e una maggiore libertà nelle provviste, ma si temeva una maggiore difficoltà per il reperimento dei mezzi materiali.

In questo clima di incertezza, alcuni episcopati nazionali europei, nei Paesi in cui il sistema beneficiale aveva avuto un ruolo importante per diversi secoli, iniziarono lo studio dei modi migliori per compiere il nuovo dettato della legge e approdarono a intese con le autorità civili e alla redazione di norme particolari "concordate con la Sede Apostolica e dalla medesima approvate" (can. 1272). Per trasmettere questi cambiamenti ai fedeli delle loro Chiese e spiegare loro la nuova situazione, i Vescovi riuniti in Conferenza episcopale emanarono documenti di diverso tipo. Nella riflessione portata avanti per redigere questi documenti si partiva dalla recentemente approfondita prospettiva ecclesiologica della comunione e, in alcuni casi, si faceva esplicito riferimento alla nozione di "corresponsabilità" di tutti i fedeli. L'esempio più evidente è il documento dell'episcopato italiano pubblicato nel 1988 sotto il titolo "Sovvenire alle necessità della Chiesa. Corresponsabilità e partecipazione dei fedeli" ${ }^{35}$. Poco posteriore, ma comunque previo al documento dei vescovi americani e riportante anche la parola "corresponsabilità" nel titolo, è il documento della

${ }^{34}$ Il tributo diocesano "ordinario" è comunque poco usato nella prassi pastorale diocesana. Tuttavia, esso possiede dei tratti molto interessanti dal punto di vista giuridico, non soltanto perché conferma la potestà tributaria della Chiesa, ma anche perché costituisce uno strumento di redistribuzione della ricchezza tra i diversi istituti diocesani che agevola certamente l'affermarsi della giustizia (cfr. J. MiÑAmBREs, Il tributo diocesano ordinario come strumento di governo, in: A. Cattaneo [cur.], L'esercizio dell'autorità nella Chiesa. Riflessioni a partire dall'Esortazione apostolica "Pastores gregis", Venezia 2005, p. 121-135; anche in Ius Ecclesiae 16[2004] 619-637).

${ }^{35}$ Notiziario CEI (1988) n. 7, p. 157-186. Il documento è stato ricordato e riproposto nel 2008 con una lettera dell'Episcopato nel ventesimo anniversario dell'avvio del nuovo sistema di sostegno economico alla Chiesa cattolica in Italia: Sostenere la Chiesa per servire tutti. A vent'anni da "Sovvenire alle necessità della Chiesa", 4 ottobre 2008. Entrambi sono reperibili in internet. Cfr. anche l'interessante commento di M. Rivella, Buon governo e corresponsabilità, Ius Ecclesiae 24(2012) 293-302, che si richiama espressamente all'idea di "stewardship". 
Conferenza episcopale spagnola su laici, Chiesa e mondo del $1991^{36}$. Per la verità, i Vescovi spagnoli partono da una prospettiva diversa da quella dei vescovi italiani e di quegli americani che non riguarda la corresponsabilità di tutti i fedeli ma si riferisce soltanto ai laici, e non si concentra sulle responsabilità economiche ma esamina le possibilità di "azione" del laico nella Chiesa e nel mondo.

\section{Due premesse per la comprensione della "stewardship"}

Prima di passare finalmente agli aspetti concreti del finanziamento pubblico delle confessioni in senso proprio, appare giusto fare un breve cenno a due aspetti della comunione ecclesiale che potrebbero giovarsi del modo di capire la corresponsabilità che è stato esplicitato nei documenti dei Vescovi richiamati, in particolare in quello americano, e che possono anche influenzare il modo di comprendere il finanziamento della confessione cattolica: si tratta della partecipazione dei fedeli ai processi decisionali nella Chiesa e della comprensione del ruolo specifico dei fedeli laici nella comunione.

Per quanto riguarda il primo pin $^{37}$ la prospettiva della stewardship può aiutare a delimitare meglio il significato della collaborazione negli organi collettivi dell'organizzazione ecclesiastica da parte dei fedeli in essi coinvolti, soprattutto in quei consigli auspicati dal Concilio Vaticano II e confermati dalle norme del Codice che mirano ad aiutare l'azione di governo ${ }^{38}$. In particolare, serve a comprendere con pienezza

${ }^{36}$ Conferencia Episcopal Española, Los cristianos laicos, Iglesia en el mundo. «Id también vosotros a mi viña» (Mt 20,4). Líneas de acción y propuestas para promover la corresponsabilidad y participación de los laicos en la vida de la Iglesia y en la sociedad civil, 19 novembre 1991, in www.conferenciaepiscopal.es.

${ }^{37}$ L'argomento è stato molto studiato da diversi angolature: cfr., ad esempio, M. RIVElla (ed.), Partecipazione e corresponsabilità nella Chiesa. I Consigli diocesani e parrocchiali, Milano 2000; e P. GHERRI (ed.), Responsabilità ecclesiale, corresponsabilità e rappresentanza, Città del Vaticano 2010. È stato anche oggetto di studio dell'ultima riunione del Gruppo di ricerca CASE, tenutasi a Roma il 30 ottobre 2013: "Partecipazione dei fedeli al governo della Chiesa in materia di beni temporali" (alcuni degli interventi possono essere consultati in www.casestewardship.org).

${ }^{38}$ In un contesto più ampio, in riferimento alle tematiche dello "ius publicum ecclesiasticum", ma con applicazioni alla situazione svizzera attuale, ha trattato questo 
di significato giuridico ed ecclesiologico la presenza di fedeli "esperti" nei Consigli per gli affari economici delle diocesi e delle parrocchie e nei Consigli pastorali diocesani e parrocchiali ${ }^{39}$. La partecipazione a questi organismi verrà vista come espressione della propria condizione di fedele, non come una "promozione del laicato", e assicurerà una visione più ampia dei problemi da affrontare. Nel tempo stesso, da parte degli organi gerarchici che si devono avvalere della consulenza di questi organismi, il loro intervento sarà visto come un vero aiuto al compimento della propria funzione di governo e non come mero "adempimento formale" o come un controllo esterno. Potranno così essere facilmente superati approcci parziali o deviati a questi organismi consultivi, e si troverà riaffermato il ruolo specifico di ciascuno al loro interno: la responsabilità determinante del parroco o del vescovo

argomento J.I. ARrIETA, Struttura sacramentale e configurazione sociale della Chiesa. Brevi considerazioni sulla situazione svizzera, in: L. Gerosa (a cura di), Chiesa Cattolica e Stato in Svizzera, Locarno 2009, p. 357-367.

${ }^{39} \mathrm{Cfr}$., tra gli altri, D. Le Tourneau, Les conseils pour les affaires économiques: origine, nature, Il diritto ecclesiastico 99(1988) 609-627; M. CALVI, Il consiglio parrocchiale per gli affari economici, Quaderni di diritto ecclesiale 1(1988) 20-33; F. Coccopalmerio, La "consultività" del Consiglio pastorale parrocchiale e del Consiglio per gli affari economici della parrocchia (cc. 536-537), Quaderni di diritto ecclesiale 1(1988) 60-65; S. Berlingò, I consigli pastorali, in: AA. VV., La Synodalité. La participation au gouvernement dans l’Église, in : «L'année canonique», hors série, vol. II (1990) 717-744; H. MülLER, Comunione ecclesiale e strutture di corresponsabilità: dal Vaticano II al Codice di diritto canonico, in: AA. VV., Comunione ecclesiale e strutture di corresponsabilità, Roma 1990, p. 17-35; J.I. ArRIETA, Órganos de participación y corresponsabilidad en la Iglesia diocesana, Ius Canonicum 34(1994) 553593; A. VizzArri, Il consiglio diocesano per gli affari economici, Monitor ecclesiasticus 119(1994) 269 ss. e 385-432; P.G. MARCUZzI, Il consiglio pastorale parrocchiale, in: AA. VV., Ius in vita et in missione Ecclesiae (Acta Symposii internationalis iuris canonici, 19-24 aprilis 1993), Città del Vaticano 1994, p. 437-463; V. DE PAolis, Il Consiglio parrocchiale per gli affari economici e $i$ beni patrimoniali della parrocchia, in: AA. VV., La parrocchia, Città del Vaticano 1997, p. 267-288; M. Rivella (ed.), Partecipazione e corresponsabilità nella Chiesa. I Consigli diocesani e parrocchiali, Milano 2000; B. UGGÈ, Il "munus regendi" dei laici in parrocchia, Quaderni di diritto ecclesiale 17(2004) 413-438; C. BEGUs, Responsabilità patrimoniale degli Organi canonici di consultazione, in: P. Gherri (ed.), Responsabilità ecclesiale, corresponsabilità e rappresentanza, Città del Vaticano 2010, p. 183-200. 
nella decisione finale, la collaborazione leale con gli uffici episcopali e parrocchiali di governo, ecc.; e sarà adeguata la rappresentatività degli istituti alla loro natura propria ${ }^{40}$, senza forzature che snaturerebbero, appunto, il loro contributo.

L'altro aspetto su cui riflettere in chiave di stewardship nel contesto della corresponsabilità di tutti i fedeli per il sostegno economico è di natura costituzionale e riguarda il ruolo dei laici nella Chiesa. Come esortava il decreto Apostolicam actuositatem ${ }^{41}$, «l'impegno d'informare dello spirito cristiano la mentalità e i costumi, le leggi e le strutture della comunità in cui uno vive, è compito e obbligo dei laici così che non può mai essere debitamente assolto dagli altri» (n. 13a). Come ricorderà il documento dei vescovi americani, la fedeltà alla chiamata di ciascuno dei fedeli all'interno della Chiesa si vive in ogni momento della propria vita e in ogni attività che si svolge in qualsiasi ambito ci si venga a trovare. Ognuno sa di dover rispondere come discepolo alle grazie ricevute dal cielo nella propria vita, senza necessità di "imitare" le risposte degli altri. La vocazione cristiana si esplica attraverso i diversi carismi e le loro attuazioni pratiche individuali e istituzionali. E la collaborazione di ciascuno, anche se da diverse situazioni soggettive e manifestata in modi differenti, è imprescindibile per la comunione. Perciò, da questa prospettiva, il ruolo dei fedeli laici caratterizzato dalla secolarità ${ }^{42}$ è necessario per la costruzione della comunione e, quindi, sarebbe deleterio cercare di eliminarlo per rendere i laici più somiglianti ai chierici o ai religiosi ${ }^{43}$.

\footnotetext{
${ }^{40} \mathrm{Su}$ questo argomento sono interessanti i rilievi di J.I. ArRIETA, Organos de participación y corresponsabilidad en la Iglesia diocesana, Ius Canonicum 34(1994) 569-574.

${ }^{41}$ Cfr. AAS 58 (1966) 837-864.

${ }^{42} \mathrm{Cfr}$. Christifideles laici, n. 15. L'argomento continua ad interessare la canonistica come dimostra la recente organizzazione di convegni sulla materia: cfr. da ultimo, L. Navarro - F. Puig, Il fedele laico. Realtà e prospettive, Milano 2012.

${ }^{43}$ Benedetto XVI ha richiamato questa idea: « La corresponsabilità esige un cambiamento di mentalità riguardante, in particolare, il ruolo dei laici nella Chiesa, che vanno considerati non come «collaboratori» del clero, ma come persone realmente «corresponsabili» dell'essere e dell'agire della Chiesa. E' importante, pertanto, che si consolidi un laicato maturo ed impegnato, capace di dare il proprio specifico contributo alla missione ecclesiale, nel rispetto dei ministeri e dei compiti che ciascuno ha
} 


\section{La "stewardship" nel contesto del sostegno economico pubblico alla Chiesa}

Passiamo adesso agli aspetti più direttamente attinenti al finanziamento della Chiesa. Secondo quanto prima spiegato, la comprensione della corresponsabilità come manifestazione della comunione in chiave familiare (derivata dallo studio del documento sulla stewardship dei vescovi americani e degli altri documenti episcopali contemporanei) fornisce il quadro per lo studio degli strumenti giuridici, organizzativi e pastorali che consentono un approccio equilibrato all'argomento del finanziamento delle attività necessarie per svolgere la missione della Chiesa (delle Chiese), anche in collaborazione con le autorità civili. Non potrò fare in questa sede riferimenti specifici alla situazione concreta dei rapporti tra confessioni e autorità civili nelle diverse sedi europee; mi limiterò a esplorare delle possibilità e delle prospettive con qualche riferimento meramente esemplificativo.

Se riandiamo al documento americano che guida queste nostre riflessioni, i vescovi ricordano ai fedeli cattolici la loro responsabilità nel seguire Cristo e poi cercano di esemplificare mediante il ricorso alle tre " $\mathrm{t}$ ": time, talent and treasure; tempo, talento e soldi. Si passa così ad una dimensione più "misurabile" della stewardship, quella che riguarda l'impegno concreto di ciascuno nelle attività che si rifanno alla Chiesa attraverso un coinvolgimento creativo o propositivo, oppure con la propria presenza o mediante l'offerta di beni. Certo, questo sviluppo comporta dei rischi di eccessiva misurazione statistica della "conversione" o della coerenza di vita cristiana. Così alcuni degli studi sulla stewardship pubblicati negli Stati Uniti possono arrivare ad oscurare quello che volevano mostrare, a far pensare cioè che quello che veramente conta sono i miei contributi "misurabili" (ore di presenza in chiesa, soldi offerti, idee avute, ecc.) e non la mia sequela di Cristo

nella vita della Chiesa e sempre in cordiale comunione con i Vescovi » (BenEDEtTo XVI, Messaggio in occasione della VI assemblea ordinaria del Forum Internazionale di Azione Cattolica, 10 agosto 2012, in www.vatican.va/holy_father/benedict_xvi/ messages/pont-messages/2012/documents/hf_ben-xvi_mes_20120810_fiac_it.html). 
in ogni circostanza ${ }^{44}$. Le misurazioni statistiche non potranno mai mostrare l'impegno cristiano dei fedeli: è impossibile "contare", ad esempio, il contributo alla comunione apportato da un malato terminale alla propria comunità parrocchiale o diocesana; oppure, con riferimento a eventi meno tragici e più frequenti, è altrettanto impossibile stabilire quanto contribuisce alla comunione effettiva della Chiesa una madre di cinque figli piccoli che riesce con difficoltà ad andare a Messa la domenica.

Ma indubbiamente, una certa misurazione dell'azione pastorale è importante ${ }^{45}$. Per questo motivo, e con la consapevolezza dei rischi insiti nella scelta, il documento episcopale americano chiede ai fedeli di misurare la responsabilità personale nel contributo alla comunione attorno ai tre concetti sottolineati (le tre " $t$ "): talento, tempo e denaro. $E$ qui si ottiene un primo effetto di finanziamento delle attività necessarie per la missione della Chiesa che potremmo qualificare da intra-ecclesiale: i fedeli, resi più consapevoli, anche attraverso i numeri, dell'importanza della loro partecipazione, aumentano il loro coinvolgimento nella comunione della Chiesa e la loro generosità in termini di dedicazione del proprio talento, del proprio tempo e dei propri soldi.

Il prof. Charles Zech, della Villanova University in Pensylvania, vicino a Philadelphia (USA), lamentava, in uno dei suoi libri, che i cattolici americani sono meno generosi dei loro concittadini protestanti ${ }^{46}$. La "pastoral letter" della conferenza dei vescovi puntava esplicitamen-

${ }^{44}$ Cfr., ad es., l'interessantissimo libro di Charles ZECH, Best Practices in Parish Stewardship, Huntington, Indiana, 2008 che, comunque, a nostro avviso, dà eccessivo rilievo ai dati statistici.

${ }^{45}$ Interessante in questo senso l'intervento alla Giornata di studio del Gruppo CASE (24 ottobre 2012) del pastoralista A. GRANADOs, La comunicazione del progetto pastorale come via per stimolare la corresponsabilità ecclesiale, in www.casestewardship. org.

${ }^{46}$ «Most Catholics are surprised to learn that, compared to their Protestant friends, they rank very low in terms of their support for their Church. (...) Most studies have found that the typical Protestant household contributes in the range of 2 to $2.4 \%$ of their income to their Church, while Catholic households contribute from 1 to $1.2 \%$ » (C. ZECH, Best Practices in Parish Stewardship, Huntington, Indiana, 2008, "Introduction"). 
te a capovolgere questa tendenza. E anche se non ci sono statistiche generali che misurino la risposta dei fedeli, dopo vent'anni dalla pubblicazione della "lettera pastorale" vi sono delle iniziative che dicono di constatare un miglioramento evidente della situazione finanziaria delle comunità che assumono consapevolmente uno stile di vita ispirato alla "stewardship" (diverse diocesi e parrocchie proclamano le conquiste fatte in questo terreno sulle pagine web dell'International Catholic Stewardship Council ${ }^{47}$, o su quelle della Leadership National Roundtable on Church Management ${ }^{48}$, che ha anche indetto un premio annuale per l'istituzione che riesce a mettere meglio in pratica le sue "Best Practices"). A mio avviso, 1'approccio al sostegno della Chiesa che si rivolge in primo luogo ai propri fedeli non deve essere trascurato, e penso che la "stewardship" offra un valido punto di partenza per questo approccio basilare.

Ma ora dobbiamo affrontare le ricadute di questa visione sul finanziamento "pubblico" delle confessioni religiose, e in particolare della Chiesa cattolica in Europa. Il ragionamento deve prendere le mosse dalla consapevolezza da parte dei singoli fedeli e dell'intera comunità di essere destinatari di molti e grandi doni. Tra questi doni ricevuti, che secondo il dettato evangelico devono essere messi a frutto ${ }^{49}$ amministrandoli rettamente, vi è certamente l'appartenenza ad una comunità sociale (che ha poi una determinata sistemazione politica, una tradizione culturale, delle espressioni artistiche e linguistiche, ecc.). Un dono che determina la stessa esistenza umana che non è tale, umana appunto, se non sviluppa la sua dimensione sociale ${ }^{50}$. Questo dono in prospettiva di stewardship è percepito come qualcosa da mettere a servizio

\footnotetext{
${ }^{47}$ Cfr. catholicstewardship.com.

${ }^{48}$ Cfr. www.theleadershiproundtable.org.

${ }^{49}$ Vedi, ad es., Lc 3, 9.

${ }^{50}$ Evidentemente, non possiamo affrontare qui in modo conveniente lo studio di questa realtà; accenniamo soltanto alla "essenzialità" della socialità umana. Vi sono molti lavori su questo argomento da parte di sociologi (cfr., ad es., P. DonATI, Il paradigma relazionale nelle scienze sociali. Le prospettive sociologiche, Bologna 2006), di filosofi e anche di teologi (cfr. tra gli altri, J. RATZINGER, Introduzione al cristianesimo, Brescia 1969).
} 
dell'intera comunità. Il cristiano corresponsabile cerca di aiutare i suoi concittadini in tutti gli aspetti della loro vita. Allo stesso tempo, perché consapevole della necessità del proprio contributo, riconosce l'arricchimento personale che ottiene dagli altri e lo trasforma a sua volta in qualcosa per la quale rendere grazie e da far fruttare. Si ingenera così una specie di circolo della corresponsabilità che comporta la generosità nel contributo al bene degli altri e l'esigenza di ricevere quello che spetta a ciascuno: più si dona e più si riceve, e più si riceve e più si dona. Questo meccanismo, che dovrebbe funzionare in ogni organizzazione sociale nella quale i membri volessero migliorarsi, nella Chiesa viene richiesto dalla sua essenziale configurazione comunionale e dalla chiamata alla santità di tutti i suoi fedeli. In relazione al finanziamento pubblico delle confessioni apporta una visione di responsabilità civica da parte dei fedeli che contribuisce profondamente ad un atteggiamento positivo riguardo al sostegno economico delle istanze che possono garantire la realizzazione del "fatto religioso" in generale e del culto a Dio in particolare.

In termini di finanziamento, la corresponsabilità si manifesterà tanto nel contributo di ciascuno al funzionamento della Chiesa quanto nel richiedere agli altri componenti della cittadinanza di riconoscere gli apporti della Chiesa al benessere sociale e di finanziarli nella misura che si ritenga giusta. La dimensione religiosa della persona umana ha sempre delle manifestazioni, sia individuali che collettive, nel contesto sociale (se si allarga la riflessione all'intera storia dell'umanità si vede come tale dimensione possa arrivare perfino a manifestazioni perverse, come il culto al diavolo, i sacrifici umani ed altre). Ogni società organizzata si trova a dover "filtrare" in qualche modo tali manifestazioni per individuare quelle che sono positive dalle altre che apportano soltanto problematicità sociale. Le manifestazioni che ho denominato "positive" contribuiscono al benessere dei singoli cittadini e della comunità come tale. In questo senso, tali manifestazioni "positive" della religiosità acquistano un qualche titolo ad essere protette e, addirittura, promosse. Uno dei modi di proteggere e di promuovere il fatto religioso è il finanziamento delle attività che rendono possibile il suo 
avverarsi: il culto, gli altri lavori dei diversi ministri, le attività di assistenza e di carità, l'educazione, ecc. Certamente, gli strumenti tecnici che permetteranno il finanziamento di queste attività saranno diversi secondo i casi (dalle esenzioni fiscali a veri e propri contributi), ma la "responsabilità" dei fedeli consci della propria chiamata alla santità li porterà a battersi perché la compagine sociale nella quale vivono e alla quale contribuiscono si dia da fare per facilitare anche le esigenze religiose individuali e collettive ${ }^{51}$.

La giustificazione tradizionale per il finanziamento pubblico delle confessioni religiose, che partiva dal fatto che lo Stato, in diversi momenti della sua storia, aveva incamerato in modo più o meno illecito i beni ecclesiastici e, quindi, che veniva a configurare il finanziamento stesso come una compensazioni per i danni arrecati in passato, sembra non reggere più. La memoria storica delle società è breve, e protrarre ancora il ricordo delle offese dei secoli passati è stato spesso causa di conflitti. L'altra giustificazione, anch'essa tradizionale, che poggia sul riconoscimento di una "religione di Stato" ripugna oggi alla sensibilità dei più, anche se formalmente è ancora presente in diversi Paesi europei, non ultimo la Gran Bretagna.

Quindi, mi pare che la logica "dono-ringraziamento", tipica dell'idea di stewardship, possa costituire una base solida per una più moderna giustificazione degli interventi delle società organizzate (dai piccoli municipi alle unioni di Stati) nel finanziamento del fatto religioso. La laicità dello Stato non può consistere nell'ignorare il fatto religioso presente nella società, forma di "laicismo di Stato" che riporterebbe le società più sviluppate a stadi molto arretrati, ma deve manifestarsi nel

\footnotetext{
${ }^{51}$ In una società multireligiosa potrebbe essere prospettata la possibilità di assegnare fondi comuni (statali, europei, comunali, ecc.) al soddisfacimento del "fatto religioso", o della vita religiosa dei propri cittadini, alla stregua di quanto si fa per migliorare le condizioni sanitarie o educative, e dividere poi tali fondi tra i soggetti capaci di erogare attività religiose o cultuali, principalmente le confessioni religiose. La "laicità" dello Stato o della società organizzata di riferimento (infra o supra statale) verrebbe manifestata dalla trasparenza e la giustizia nell'assegnazione di quei fondi comuni, ma soprattutto dal fatto che mostrerebbe che chi gestisce la "cosa pubblica" ritiene la dimensione religiosa della vita umana una dimensione fondamentale da garantire.
} 
trattare con giustizia le esigenze di religiosità connaturali all'uomo, sia nella determinazione delle manifestazioni "positive" di tale religiosità, sia nel trattare le singole manifestazioni, anche dal punto di vista economico, in modo adeguato al loro essere e alla loro rilevanza sociale.

Promuovere la stewardship nella Chiesa contribuisce a formare cittadini con maggiore responsabilità civica, consapevoli della personale responsabilità di contribuire al bene comune e, in quanto cattolici, al culto a Dio che richiede ogni vita umana per essere vissuta in pienezza. Tale consapevolezza porta a maggiore impegno in tutte le attività che riguardano il bene comune e il bene pubblico e, in particolare, per quanto adesso ci interessa, a migliorare la redditività degli sforzi fatti per favorire la realizzazione del diritto a rendere culto a Dio individualmente e collettivamente. In quest'ottica, il cattolico corresponsabile facilita il sostegno alle confessioni religiose in tutte le forme, anche attraverso i contributi pubblici.

\section{Conclusione}

In conclusione, la stewardship predicata dai vescovi americani nel documento che abbiamo presentato porta a che ciascuno prenda su di sé la propria parte nella costruzione della Chiesa. Presa di coscienza che si riflette nella generosità per venire incontro alle necessità della Chiesa (l'obbligo-diritto di tutti i fedeli sancito dal can. $222 \S 1$ $\mathrm{CIC}^{52}$ ), ma anche nell'iniziativa apostolica di cui al can. $216 \mathrm{CIC}^{53} \mathrm{e}$ nella partecipazione all'azione di governo ed in generale all'impostazione dell'azione evangelizzatrice e missionaria secondo la scienza, la competenza ed il prestigio di ciascuno, a norma del can. $212 \S 3 \mathrm{CIC}^{54}$.

${ }^{52}$ « I fedeli sono tenuti all’obbligo di sovvenire alle necessità della Chiesa, affinché essa possa disporre di quanto è necessario per il culto divino, per le opere di apostolato e di carità e per l'onesto sostentamento dei ministri ».

${ }^{53}$ « Tutti i fedeli, in quanto partecipano alla missione della Chiesa, hanno il diritto, secondo lo stato e la condizione di ciascuno, di promuovere o di sostenere l'attività apostolica anche con proprie iniziative; tuttavia nessuna iniziativa rivendichi per se stessa il nome di cattolica, senza il consenso dell'autorità ecclesiastica competente ».

${ }^{54}$ « In modo proporzionato alla scienza, alla competenza e al prestigio di cui godono, essi hanno il diritto, e anzi talvolta anche il dovere, di manifestare ai sacri Pastori 
Così, il fedele corresponsabile, tra le altre cose, contribuisce in diversi modi ad una gestione trasparente dei beni della Chiesa che rende anche chiaro ai non fedeli l'uso dei beni ecclesiastici.

Oltre a questa azione "nascosta" della stewardship, vi è poi il diretto coinvolgimento del fedele corresponsabile nel proprio contesto sociale che lo porta a lottare perché la dimensione religiosa della persona umana e delle società venga esplicitamente riconosciuta anche attraverso il finanziamento delle attività rivolte a sostenere tale dimensione; a promuovere il bene comune della società in cui vive apportando attivamente il suo contributo; ad assumersi la propria responsabilità apostolica ed evangelizzatrice in modo personale, senza addossarla sempre ad altri soggetti; ecc. Un cittadino così contribuisce sicuramente al miglioramento della società alla quale appartiene e farà fruttare al massimo tutte le risorse disponibili, anche quelle destinate al finanziamento dei culti.

Quindi, per rispondere al punto interrogativo riportato nel titolo di questo lavoro, l'idea americana di stewardship può essere importata in Europa, con i convenienti adattamenti, e può interagire perfettamente con i diversi sistemi di sostegno alle confessioni religiose da parte delle autorità civili. Il processo di adattamento richiede approfondimento e riflessione non soltanto sugli aspetti "tecnici" dei diversi sistemi di finanziamento ma anche sulle basi costituzionali del diritto di libertà religiosa. Tuttavia, tale processo sembra prospettarsi salutare, sia per le singole confessioni (almeno, per quella cattolica), sia per i diversi soggetti sociali.

\section{Stewardship, administration and economic sustenance for the Church: Towards a European stewardship?}

Baptism brings with it the sharing of the vision and the mission of the Church, the stewardship that springs from conversion. The knowledge of this basic theological reality manifests itself in the commitment to provide for the

il loro pensiero su ciò che riguarda il bene della Chiesa; e di renderlo noto agli altri fedeli, salva restando l'integrità della fede e dei costumi e il rispetto verso i Pastori, tenendo inoltre presente l'utilità comune e la dignità della persona ». 
needs of the community. The Catholic Conference of US bishops specificied the economic facets of this commitment as giving time, talent and treasure. This vision could be tried in other communities outside of the Church (political communities) and could offer a new perspective for basing the need to finance religious confessions.

PAROLE ChIAVE: Corresponsabilità, sovvenire alle necessità delle Chiesa, libertà religiosa

KEY WORDS: stewardship, provide for the needs of the Church, religious freedom 